ITC 1/48

Journal of Information Technology and Control

Vol. 48/ No.1/ 2019

pp. 146-159

DOI 10.5755/j01.itc.48.1.20162
Robust Fault Diagnosis for Discrete-Time Switched System with Unknown State Delays Subject to Component Faults
Accepted after revision 2019/01/22
Received 2018/07/12

\title{
Robust Fault Diagnosis for Discrete-Time Switched System with Unknown State Delays Subject to Component Faults
}

\section{Haigang Zhang}

Tianjin Key Lab for Advanced Signal Processing; Civil Aviation University of China; Tianjin Dongli District, Tianjin North Highway 2898, China; e-mail: zhg2018@sina.com

Corresponding author: zhg2018@sina.com

This paper investigates the problems of fault detection and diagnosis in a class of discrete-time switched systems with unknown state delay against component faults. The component fault, also called the multiplicative fault or loss of actuator effectiveness, is a common type of operation fault for switched systems, which has been paid more attention. In this paper, the learning observers (LOs) technology are proposed to reconstruct both the system state and component faults. The proposed LOs technique is also extended to the system with unknown time delay and external disturbance. The sufficient conditions for the convergence of the output of LOs and the uniformly ultimately boundedness of the reconstructed errors are proved by the switched Lyapunov function and $H_{\infty}$ technique. The proposed fault reconstruction strategy has certain tolerance for fault types, which is effective for both slow and fast time-varying faults by adjusting the iteration step size. In the simulation parts, three kinds of fault signals are taken into consideration to verify the effectiveness and robustness of the proposed method.

KEYWORDS: Fault Diagnosis, Switched System, Unknown State Delay, Lyapunov Function.

\section{Introduction}

With the increasing complexity of modern industrial systems and higher pursuit of system performance, fault diagnosis is an important and challenging problem in many practical systems, such as chemical en-

gineering, nuclear engineering and aerospace engineering $[9,12]$. Faults may occur at any time, and then destroy the system stability or even make the system tend to collapse. Nowadays, fruitful model-based fault 
diagnosis works have been published in several excellent papers and books $[3,5,14,15,17,23,26]$. Among these approaches, the most commonly used one is first to design the observers or filters to reconstruct the fault signal or residual signal, and then generate an alarm when the residual function is larger than the threshold or the fault reconstruction signal is not zero [18].

Recently, several physical systems present hybrid behaviors $[6,19]$. Wherein, the switched systems play an important role in industrial process and have drawn more and more attention for the stability and controllability research [5]. A generalized switched system is composed of subsystems with their own parameters subject to a specific rule orchestrating the switching law among the various subsystems. The motivation of studying switched systems drives from the facts that many physical scenes exhibit the switching features among multi-models.

Because of the increasing demand for the operational safety refer to switched systems, the research about fault diagnosis of switched systems has been a hot topic [7]. Hwang et al. [12] introduced three types of fault in the field of model-based systems, including actuator fault, sensor fault and component fault or loss of effectiveness fault. The actuator fault and sensor fault are also called the additive fault, because they have an additional relationship with the original system state. There are many excellent fault diagnosis methods for additive faults in switched systems. In [4], a multiple Lyapunov function approach was employed for fault detection and diagnosis in switched systems. Then the switched Lyapunov function approach was first applied in stability analysis of switched systems [7]. Wang et al. [20] designed a fault filter for the actuator fault and sensor fault based on $H_{\infty}$ performance index. Hespanha et al. [11] applied the average dwell time technology to conduct stability analysis for switched systems, while Zhang et al. [23] converted the fault detection problems into an $H_{\infty}$ filtering problem by using the average dwell time technology. Du et al. [8] adopted the asynchronous switched approach in switched systems to reconstruct the additive faults.

Compared with the actuator and sensor faults, the component faults or loss of effectiveness faults can also be called the multiplicative faults, which can weaken system input performance, and destroy the stability. It is necessary to design fault diagnosis strategy for component faults in switched systems. There are relatively fewer research results for the switched systems with component faults. He et al. [10] designed a fault detection filter for a more general sensor fault, including the loss of effectiveness, the outage and the drifted fault in switched systems. The effectiveness of sensor was decayed in a certain proportion, and the attenuation rate was assumed to be a constant. Rodrigues et al. [16] proposed a fault tolerant control model for switched systems with component faults. However, the fault signal was set to be constant or slow time-varying. The component faults are multiplied with the actuator and sensor, which is the main reason why it is difficult to design the fault diagnosis method. In this paper, the learning observer technology is applied to make reconstruction for the component faults in switched systems, which is a new attempt.

As is well-known, time-delay phenomenon is very common in many real physical systems, such as air pollution system and chemical process etc. [23]. It would often cause the instability and poor performance of the switched systems. There are some works about fault diagnosis taking time-delay phenomenon into consideration [20,22]. Wang et al. [20] proposed a robust fault detection method for switched linear systems with state delay. Yu et al. [22] presented a fault detection frame for discrete-time switched systems with distributed delays. However, the existing models were only for additive faults. In addition, the delay time was known in advance. It should be mentioned that in our previous work [25], we designed an adaptive fault diagnosis model for continuous time-delay repetitive systems subject to sensor fault, where the delay time was known in advance. In this paper, the unknown time-delay element is taken into consideration. A relatively slack restriction for delay time with known upper and lower bounds improves the generalization performance of fault reconstruction model.

In this work, a fault diagnosis method based on LOs against the component faults for switched systems is proposed. It can handle the task of fault diagnosis for more complex systems, even with unknown delay time and external disturbance. Compared with the existing works for the constant faults or slowly time-varying faults, the proposed method is effective for relatively fast time-varying faults. By using the switched Lyapunov function and $H_{\infty}$ technique, the sufficient conditions for the convergence of the output of LOs are presented in term of linear matrix 
inequalities (LMIs). Finally, the simulation results for three forms of loss of actuator effectiveness faults demonstrate the feasibility of this scheme. To simplify, the contributions of this paper can be summarized as follows:

1 The LOs are employed in switched systems against component faults, which is a new attempt.

2 The time-delay element and external disturbance are taken into consideration in the fault diagnosis model. A more relaxed bounded constraint for delay time is assumed, which leads to the proposed LOs having better generalization performance.

3 The proposed fault reconstruction strategy has certain tolerance for fault types. It is still valid for fast time-varying faults.

The rest of the paper is organized as follows. In Section 2, the description for a generalized switched system is presented and the problems are formulated. Section 3 is the main part of this paper, which presents the robust design of LOs. Simulation results are carried out in Section 4 and conclusions are drawn in Section 5. Section 6 is the appendix, where the proofs of the theorems are presented.

Notations: The notations used throughout the paper are normative. $A^{T}, A^{-1}, \lambda(A)$ and $\|A\|$ are the symbols to denote, respectively, the transpose, the inverse, the eigenvalues and the Euclidean norm of any square matrix $A$. Particularly, $\lambda_{\max }(A)$ and $\lambda_{\min }(A)$ present the maximum and minimum eigenvalues of $A$. We use $A>0(A<0)$ to denote that $A$ is positive (negative) defined and $I$ to denote the identity matrix with an appropriate dimension. \|\|$_{\infty}$ represents the infinity norm of any vector. The symbol * means the symmetric terms in some matrices.

\section{System Description and Problem Formulation}

We consider the following discrete-time switched system

$$
\left\{\begin{array}{rl}
x(k+1) & =A_{\sigma(k)} x(k)+B_{\sigma(k)} f(k) u(k) \\
y(k) & =C_{\sigma(k)} x(k)
\end{array},\right.
$$

where $x(k) \in R^{m}, u(k) \in R^{n}$ and $y(k) \in R^{p}$ are the state, control input and output, respectively. Diagonal matrix $f(k)=\operatorname{diag}\left\{f_{1}(k), \cdots, f_{n}(k)\right\}$ represents the component faults with $f_{i}(k) \in(0,1]$. Specifically, when $f_{i}(k)=1$, the $i$ th actuator runs fault-freely; if $0<f_{i}(k)<1$, partial effectiveness of the actuator is lost; and $f_{i}(k)=0$ means that the actuator has no effectiveness in the system totally. $\sigma$ is the switching rule which takes the values in the finite set $\aleph:\{1, \cdots, N\}$, where $N$ is the number of subsystems. It is assumed that $\sigma(k)$ is dependent on $k$ or $x_{k}$, or other switching rules at an arbitrary discrete time $k . A_{\sigma}, B_{\sigma}$, and $C_{\sigma}$ are of appropriate size constant known matrices. Since $f(k)$ is a diagonal matrix, the switched system can be rewritten as follows

$$
\left\{\begin{array}{c}
x(k+1)=A_{\sigma(k)} x(k)+B_{\sigma(k)} U_{k} F_{k} \\
y(k)=C_{\sigma(k)} x(k)
\end{array},\right.
$$

where $U_{k}=\operatorname{diag}\left\{u_{1}, \cdots, u_{n}\right\}$ and $F_{k}=\left[f_{1}, \cdots, f_{n}\right]^{T}$. The fault signals are presented by a column vector, which is suitable for the following analysis and the proofs of the convergence theorems.

Assumption 1: The switching rule $\sigma(k)$ is available at each sampling time $k$. Additionally, only one subsystem is active each time.

In most case, the switching signal is not known beforehand, and that means one can not know in advance the distribution of conversion signals. However, the instantaneous value of switching signals is available. Thus, the design of observer modes will be modified with the change of switching signals. Some similar operations are shown in $[2,20]$.

Here we introduce the switching signal function as $\xi(k)=\left[\xi_{1}(k), \cdots, \xi_{n}(k)\right]^{T}$, where $\xi_{i}(k): Z^{+} \rightarrow\{0,1\}$ and $\sum_{i=1}^{N} \xi_{i}(k)=1, k \in Z^{+} . \xi_{i}(k)=1$ means that the $i$ th subsystem is active at $k$ th sampling step, while it is out of operation when $\xi_{i}(k)=0$. Then one can rewrite the switched system as

$$
\left\{\begin{array}{rl}
x(k+1) & =\sum_{i=1}^{N} \xi_{i}(k)\left(A_{i} x(k)+B_{i} U_{k} F_{k}\right) \\
y(k) & =\sum_{i=1}^{N} \xi_{i}(k) C_{i} x(k)
\end{array} .\right.
$$


Assumption 2: The system control input $u(k)$ is norm-bounded, $\|u(k)\| \leq u_{\max }$.

Assumption 2 says that the control input is bounded, which is reasonable for a system running in the normal operating environment. Additionally, the observability is another reasonable hypothesis for the design of LOs in the following sections.

This paper aims to establish a fault diagnosis model to reconstruct the component faults $F_{k}$. The model should not only guarantee the convergence speed, but also be applied in many kinds of fault types. In addition, it should be suscesful to deal with time-dalay phenomenon and externel disturbance.

\section{Fault Reconstruction based on LOs}

\subsection{Design of Learning Observers}

In this subsection, a novel fault reconstruction method for the above switched system is presented. The corresponding LOs are established as

$$
\left\{\begin{aligned}
\hat{x}(k+1)= & \sum_{i=1}^{N}\left[\xi_{i}(k)\left(A_{i} \hat{x}(k)+B_{i} U_{k} \hat{F}_{k}\right)\right] \\
& +\sum_{i=1}^{N}[\tilde{L}(y(k)-\hat{y}(k))] \\
\hat{y}(k)= & \sum_{i=1}^{N} \xi_{i}(k) C_{i} \hat{x}(k) \\
\hat{F}_{k}= & K_{1} \hat{F}_{k-\tau}+\tilde{K}_{2}(y(k)-\hat{y}(k))
\end{aligned}\right.
$$

where $\hat{x}(k)$ and $\hat{y}(k)$ are the estimations of system state and output, respectively. $\hat{F}_{k}$ represents the reconstructed fault of loss of actuator effectiveness updated by both its previous information at the sampling step $k-\tau$ and current output error. $\tau$ is the updating interval. $K_{1}$ is a diagonal matrix, that is, $K_{1}=\operatorname{diag}\left\{k_{1}, \cdots, k_{n}\right\}$ with $k_{i} \in(0,1], i \in\{1, \cdots, n\}$, and $\tilde{L}=\sum_{i=1}^{N} \xi_{i}(k) L_{i}, \quad \tilde{K}_{2}=\sum_{i=1}^{N} \xi_{i}(k) K_{2 i} . K_{1}, \quad L_{i}$ and $K_{2 i}$ would be selected later.

Define the system state-estimation error, output-estimation error and fault-estimation error as $e_{x}(k)=x(k)-\hat{x}(k), e_{y}(k)=y(k)-\hat{y}(k) \quad$ and
$e_{F}(k)=F_{k}-\hat{F}_{k}$. In order to drive the fault reconstruction error less restrictively and reasonably, the following assumption is necessary.

Assumption 3: Denote $\tilde{F}_{k}=F_{k}-K_{1} F_{k-\tau}$ and assume $\tilde{F}_{k}$ is norm-bounded, such that $\left\|\tilde{F}_{k}\right\| \leq \kappa_{f}$.

Assumption 3 shows a relatively loose restriction compared with other works for fault diagnosis, where norm-bound condition of fault signal or its derivative is necessary. The restriction of norm-bound of the derivative of faults means that the corresponding faults are time-varying slowly, which is removed in this paper. The proposed LOs can deal with broader fault signals, including slowly and fast time-varying ones. Since $0<f_{i} \leq 1, i=1,2, \cdots, n$, the upper bound of $\|\tilde{F}(k)\|$ can be calculated easily, such as $\kappa_{f}=1+\left\|K_{1}\right\|$.

Remark 1: The choice of $K_{1}$ and $\tau$ is generally based on human experience. $K_{1}$ approximates the identity matrix with proper dimension. It presents the influence level of the historical reconstructed signal on the current one. A relatively larger value of $K_{1}$ means more information in the historical reconstructed signal is preserved, while less information is retained with relatively smaller value of $K_{1}$. In general, the value of $\tau$ is determined according to the fault signal. When dealing with the fast time-varying signal, $\tau$ should be sufficiently small. On the contrary, $\tau$ can be chosen as a larger interval if the fault signal is slow time-varying.

Define the state error $e_{x}(k)$, output error $e_{y}(k)$ and fault error $e_{F}(k)$ as

$$
\left\{\begin{array}{l}
e_{x}(k)=x(k)-\hat{x}(k) \\
e_{y}(k)=y(k)-\hat{y}(k) . \\
e_{F}(k)=F_{k}-\hat{F}_{k}
\end{array}\right.
$$

Then one can get the following dynamic equations

$$
\left\{\begin{aligned}
e_{x}(k+1)= & \sum_{i=1}^{N} \xi_{i}(k)\left[\begin{array}{l}
\left(A_{i}-L_{i} C_{i}\right) e_{x}(k) \\
+B_{i} U_{k} e_{F}(k)
\end{array}\right] \\
e_{y}(k)= & \sum_{i=1}^{N} \xi_{i}(k) C_{i} e_{x}(k) \\
e_{F}(k)= & K_{1} e_{F}(k-\tau)- \\
& \sum_{i=1}^{N} \xi_{i}(k) K_{2 i} C_{i} e_{x}(k)+\tilde{F}_{k}
\end{aligned}\right.
$$




\subsection{Parameter Configuration of LOs}

Here the analysis of LOs is presented. In order to facilitate and simplify the proof, the matrix $B_{i}$ is omitted. The convergence condition is presented in the following theorem, whose proof is shown in Part A in the appendix.

Theorem 1: Considering the switched system in (3) with Assumptions 1-3, if there exist positive definite symmetric matrices $P_{i} \in R^{m \times m}$ and $Q_{i} \in R^{n \times n}$, and matrices $K_{1}, L_{i}, K_{2 i}$, such that the following LMIs hold:

$$
\left[\begin{array}{ccc}
-\left(1+\gamma_{1}\right) P_{j}^{-1} & A_{i}-L_{i} C_{i} & 0 \\
* & -\alpha \rho_{2} I & K_{2 i} C_{i} \\
* & * & -P_{i}
\end{array}\right]<0
$$

and

$$
\alpha \rho_{1} K_{1}^{T} K_{1}-Q_{l}<0,
$$

then state estimation error $e_{x}(k)$ and fault reconstruction error $e_{F}(k)$ are uniformly bounded, where $\alpha=\left(1+1 / \gamma_{1}\right) \lambda_{\max }\left(P_{j}\right) u_{\max }^{2}+(1+\delta) \lambda_{\max }\left(Q_{l}\right) \quad$ with $\gamma_{1}>0$ and $\delta \geq 0$.

\subsection{Robust Learning Observers Design with Delay Time}

In the previous subsection, the LOs subject to the component faults are designed. Here, based on the Lyapunov stability theory and $H_{\infty}$ technique, the robust LOs are presented taking time-delay and external disturbance into consideration. The corresponding system model is

$$
\left\{\begin{array}{rl}
x(k+1) & =\sum_{i=1}^{N} \xi_{i}(k)\left(\begin{array}{l}
A_{i} x(k)+A_{d i} x\left(k-d_{k}\right) \\
+U_{k} F_{k}+E_{i} \eta_{k}
\end{array}\right) \\
y(k) & =\sum_{i=1}^{N} \xi_{i}(k) C_{i} x(k)
\end{array},\right.
$$

where $d_{k}$ represents the unknown state delay and $\eta_{k}$ is the external disturbance signal with norm boundary, $\left\|\eta_{k}\right\| \leq \bar{\eta}$. Additionally, $A_{d i}$ and $E_{i}$ are known constant matrices with appropriate dimensions.

Assumption 4: We suppose that $d_{k}$ is unknown time delay variable with known lower and upper bounds: $\underline{d} \leq d_{k} \leq \bar{d}$.

The robust LOs are designed as follows:

$$
\left\{\begin{aligned}
\hat{x}(k+1)= & \sum_{i=1}^{N}\left(A_{i} \hat{x}(k)+A_{d i} \hat{x}\left(k-d_{k}\right)+U_{k} \hat{F}_{k}\right) \\
& -\tilde{L}(y(k)-\hat{y}(k)) \\
\hat{y}(k)= & \sum_{i=1}^{N} \xi_{i}(k) C_{i} \hat{x}(k) \\
\hat{F}_{k}= & K_{1} \hat{F}_{k-\tau}+\tilde{K}_{2}(y(k)-\hat{y}(k))
\end{aligned}\right.
$$

where the symbols are defined above.

Then the state estimation error, output estimation error and fault reconstruction error are rewritten as

$$
\left\{\begin{array}{l}
e_{x}(k+1)=\sum_{i=1}^{N} \xi_{i}(k)\left[\begin{array}{l}
\left(A_{i}-L_{i} C_{i}\right) e_{x}(k)++E_{i} \eta_{k} \\
A_{d i} e_{x}\left(k-d_{k}\right)+U_{k} e_{F}(k)
\end{array}\right] \\
e_{y}(k)=\sum_{i=1}^{N} \xi_{i}(k) C_{i} e_{x}(k) \\
e_{F}(k)=K_{1} e_{F}(k-\tau)-K_{2 i} C_{i} e_{x}(k)+\tilde{F}_{k}
\end{array} .\right.
$$

The convergence condition for the robust LOs is presented in the following theorem. Its proof is shown in Part B in the appendix.

Theorem 2: Consider the switched system in (9) with Assumptions 1-4. For a given positive scalar $\gamma$, if there exist positive definite symmetric matrices $P_{i} \in R^{m \times m}, Q_{i} \in R^{m \times m}$ and $R_{i} \in R^{n \times n}$, and matrices $K_{1}$, $L_{i}, K_{2 i}, \forall i \in[1,2, \cdots, N]$, such that the following LMIs hold

$$
\begin{gathered}
{\left[\begin{array}{ccccc}
-\zeta_{1} P_{j}^{-1} & 0 & 0 & A_{i}-L_{i} C_{i} & 0 \\
* & -P_{i} & K_{2 i} C_{i} & 0 & I \\
* & * & -I & C_{i} & 0 \\
* & * & * & -\alpha \rho_{2} I & 0 \\
* & * & * & * & -\sigma Q_{i}^{-1}
\end{array}\right]<0 \quad \text { (12) }} \\
{\left[\begin{array}{cccc}
-\zeta_{2} P_{j}^{-1} & 0 & 0 & A_{d i} \\
* & -\zeta_{3} P_{j}^{-1} & A_{d i}^{T} P_{j} E & 0 \\
* & * & -\gamma^{2} I & E_{i}^{T} \\
* & * & * & -\lambda_{\min }(Q) I
\end{array}\right]<0 \quad \text { (13) }}
\end{gathered}
$$


and

$$
\alpha \rho_{1} K_{1}^{T} K_{1}-R_{l}<0,
$$

the proposed LOs can make the state estimation error, output estimation error and fault reconstruction error uniformly ultimately bounded. Additionally, the output estimation error satisfies $\left\|e_{y}(k)\right\| \leq \gamma \bar{\eta}+\sqrt{\alpha \rho_{3}} \kappa_{f}$, where $\alpha, \sigma$ and $\zeta_{i}, i=1,2,3$, would be defined in the proof.

Theorem 3: Consider the switched system in (9) with Assumptions 1-4. For a given positive scalar $\gamma$, (12) and (13) are feasible if there exist the positive $P_{i}$ and $Q_{i}$, and matrix $G_{i}, i \in \aleph$, such that the following LMIs hold:

$$
\left[\begin{array}{ccccc}
-\zeta_{1} \hat{P}_{j} & 0 & 0 & G_{i}^{T} A_{i}-Y_{i} C_{i} & 0 \\
* & -G_{i}^{T} P_{i} G_{i} & M_{i} C_{i} & 0 & G_{i}^{T} G_{i} \\
* & * & -I & C_{i} & 0 \\
* & * & * & -\alpha \rho_{2} I & 0 \\
* & * & * & * & -\sigma \hat{Q}_{i}
\end{array}\right]<0 \quad(15)
$$

and

$$
\left[\begin{array}{cccc}
-\zeta_{2} \hat{P}_{j} & 0 & 0 & G_{i}^{T} A_{d i} \\
* & -\zeta_{3} \hat{P}_{j} & G_{i}^{T} A_{d i} P_{j} E & 0 \\
* & * & -\gamma^{2} I & E_{i}^{T} \\
* & * & * & -\lambda_{\min }(Q) I
\end{array}\right]<0,
$$

where $\hat{P}_{j}=-P_{j}+\left(G_{i}+G_{i}^{T}\right), \quad \hat{P}_{j}=-P_{j}+\left(G_{i}+G_{i}^{T}\right)$, $Y_{i}=G_{i}^{T} L_{i}$ and $M_{i}=G_{i}^{T} K_{2 i}$.

Remark 2: In Part C of the appendix, the strict proof of Theorem 3 is shown. It is also the necessary condition proven in $[1,15]$. The introduction of the auxiliary slack matrix $G_{i}$ can couple the product term between $P_{j}$ and $\left(A_{i}-L_{i} C_{i}\right)$, which is less conservative for constrained problems.

\section{An Illustrative Example}

In this section, some simulation studies are presented to verify the effectiveness of the proposed LOs method. Consider the following discrete-time switched system consisting of two subsystems with parameters as

$$
\begin{aligned}
& A_{1}=\left[\begin{array}{cc}
-0.2 & 0.1 \\
0 & -0.5
\end{array}\right] \quad A_{d 1}=\left[\begin{array}{ll}
-0.1 & \\
& -0.1
\end{array}\right] \\
& B_{1}=\left[\begin{array}{cc}
1 & 0.5 \\
0.5 & 1
\end{array}\right] \quad E_{1}=\left[\begin{array}{l}
0.5 \\
0.5
\end{array}\right] \quad C_{1}=\left[\begin{array}{cc}
1 & 0.5 \\
-0.2 & 0.6
\end{array}\right] \\
& A_{2}=\left[\begin{array}{cc}
-0.3 & 0.1 \\
0.2 & -0.6
\end{array}\right] \quad A_{d 2}=\left[\begin{array}{ll}
-0.1 & \\
& -0.1
\end{array}\right] \\
& B_{2}=\left[\begin{array}{cc}
1 & 0.2 \\
0.3 & 0.5
\end{array}\right] \quad E_{2}=\left[\begin{array}{l}
0.5 \\
0.5
\end{array}\right] \quad C_{2}=\left[\begin{array}{ll}
1 & 0.5 \\
0 & 0.5
\end{array}\right] \text {. }
\end{aligned}
$$

Other parameters are chosen as $\gamma_{i}=0.5, \zeta_{i}=0.5$ with $i=1,2, \cdots, 5, \delta=0.06$ and $\gamma=1.2$. The delay time $d_{k}$ is variable, which changes with the switching signal. The upper and lower bounds of $d_{k}$ are known as $\bar{d}=6$ and $\underline{d}=2$. The system input $u(k)$ is set as

$u(k)=\left[\begin{array}{c}0.3 * \sin (k / 10) \\ 0.2 * \sin (k / 8)+0.2\end{array}\right]$.

The component fault is assumed as $F(k)=\left[\begin{array}{ll}\bar{f}(k) & \tilde{f}(k)\end{array}\right]^{T}$, where $\bar{f}(k)=1$ represents the normal actuator without fault and $\tilde{f}(k)$ stands for the loss of effectiveness fault in the second actuator component. For verifying the generalization performance of the proposed LOs, three kinds of fault modes are considered.

$\tilde{f}_{1}(k)$ represents the constant fault, which is the most common type of component faults. In general, the actuator loses the effectiveness with a fixed degree. $\tilde{f}_{1}(k)$ is defined as follows:

$$
\tilde{f}_{1}(k)=\left\{\begin{array}{ll}
0.5 & 60 \leq k \leq 120 \\
1 & \text { others }
\end{array} .\right.
$$

$\tilde{f}_{2}(k)$ is a slowly time-varying signal, which means that the actuator gradually loses its effectiveness. It will seriously affect system performance if no action exists in a timely manner. $\tilde{f}_{2}(k)$ is defined as follows:

$$
\tilde{f}_{2}(k)=\left\{\begin{array}{ll}
1-0.0005 k & 80 \leq k \leq 140 \\
1 & \text { others }
\end{array} .\right.
$$


$\tilde{f}_{3}(k)$ represents an irregular and fast time-varying fault signal, which is defined as follows:

$$
\tilde{f}_{3}(k)=\left\{\begin{array}{ll}
0.15 \sin (k / 40)+1.1 e^{-k / 3000}-0.1 & k \geq 80 \\
1 & \text { others }
\end{array} .\right.
$$

For the design of LOs, the parameter $K_{1}$ can be chosen as $0.9500 I_{2}$ and the other parameters will be calculated using Matlab LMI toolbox as follows:

$$
\begin{aligned}
& {\left[\frac{L_{1}}{K_{21}}\right]=\left[\begin{array}{cc}
0.0254 & 0.1026 \\
-0.0356 & 0.0698 \\
\hline-0.0052 & 0.0065 \\
-0.2589 & -0.3025
\end{array}\right]} \\
& {\left[\frac{L_{2}}{K_{22}}\right]=\left[\begin{array}{cc}
0.1283 & -0.1563 \\
-0.0895 & -0.0956 \\
\hline-0.0084 & 0.0105 \\
0.1986 & -0.2344
\end{array}\right] .}
\end{aligned}
$$

The switching signal is generated randomly and shown in Figure 1. The value 0 of switching signal

\section{Figure 1}

The switching signal

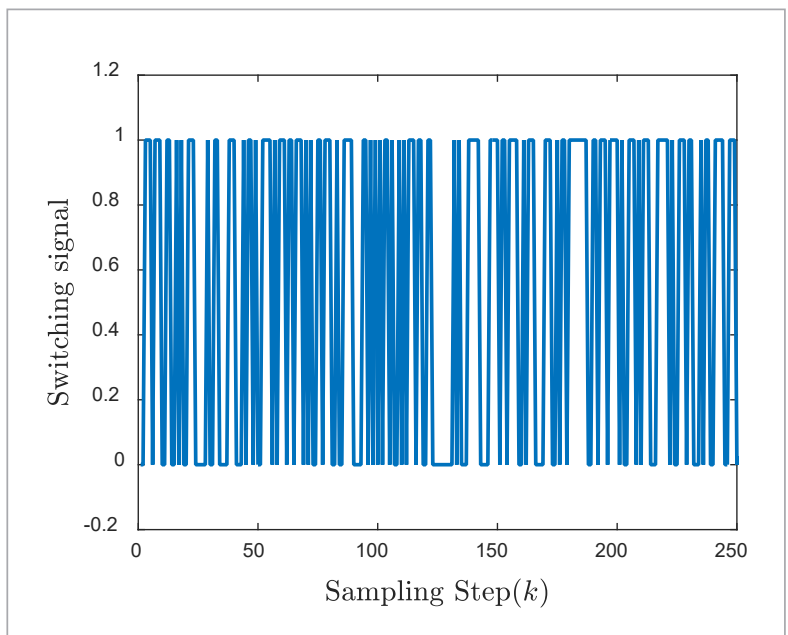

represents the first subsystem is active, while the other subsystem is on running when the switching signal is 1 . Simulation results of LOs, which refer to above three component faults, are listed in Figures 2-4. One can see that the proposed LOs can reconstruct the fault signals effectively.

\section{Figure 2}

The signals of loss control effectiveness $(\tau=6): \tilde{f}_{1}(k)$ and its estimation: $\hat{f}_{1}(k)$

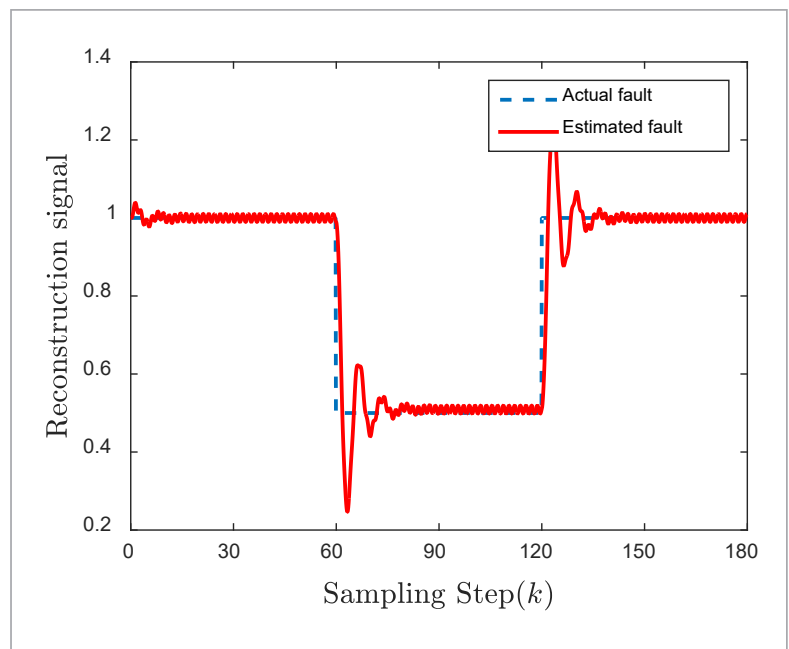

Figure 3

The signals of loss control effectiveness $(\tau=5): \tilde{f}_{2}(k)$ and its estimation: $\hat{f}_{2}(k)$

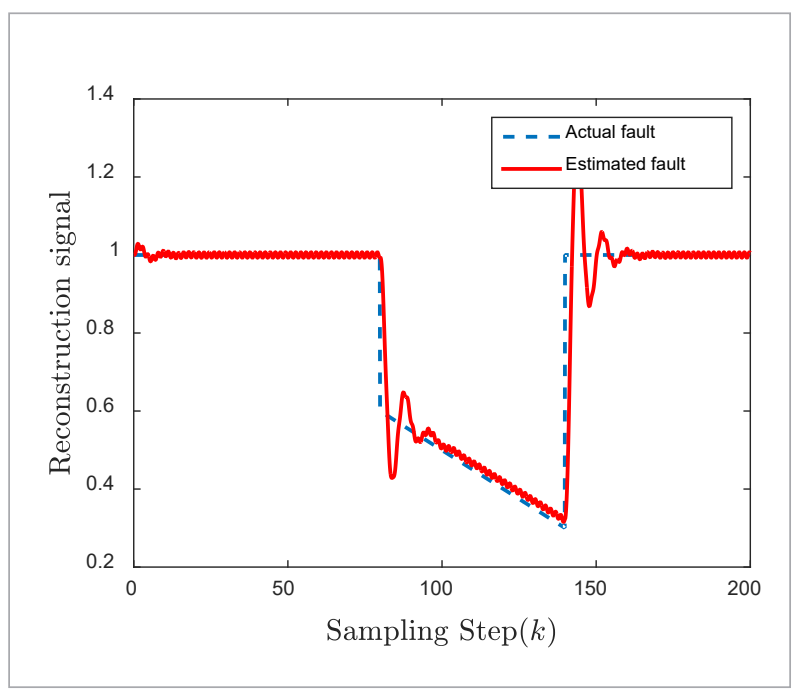

Before the occurrence of any fault, the LOs can identify the effectiveness of actuators very accurately, which means it can also be applied for fault detection applications. When the loss of actuator effectiveness happens, the LOs can reconstruct any form of faults including the constant fault in Figure 2, slowly time-varying fault in Figure 3 and fast time-varying fault in Figure 4. 
Figure 4

The signals of loss control effectiveness $(\tau=2): \tilde{f}_{3}(k)$ and its estimation: $\hat{f}_{3}(k)$

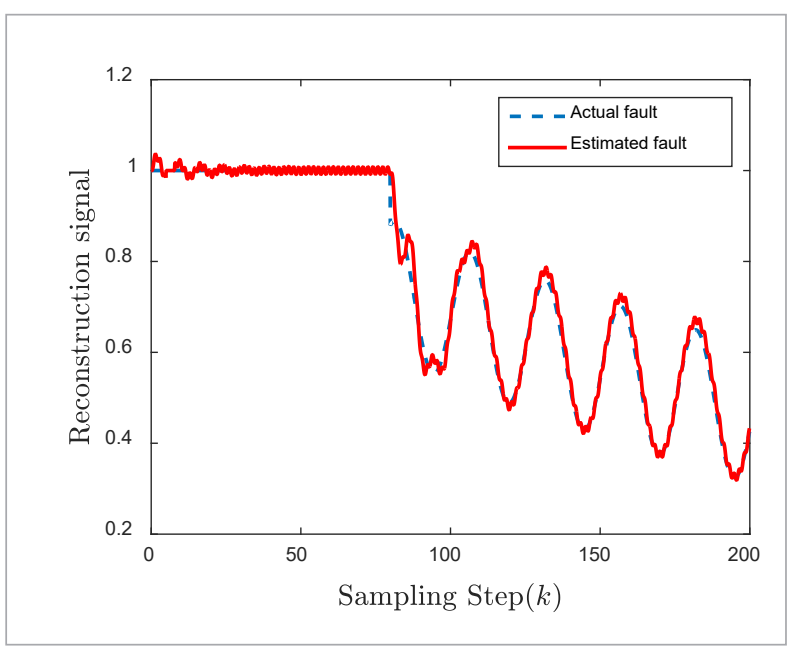

Figure 5 shows the fault reconstruction errors. The error tends to converge after small fluctuations within a limited time, which proves once again the effec-

\section{Figure 5}

The fault reconstruction errors

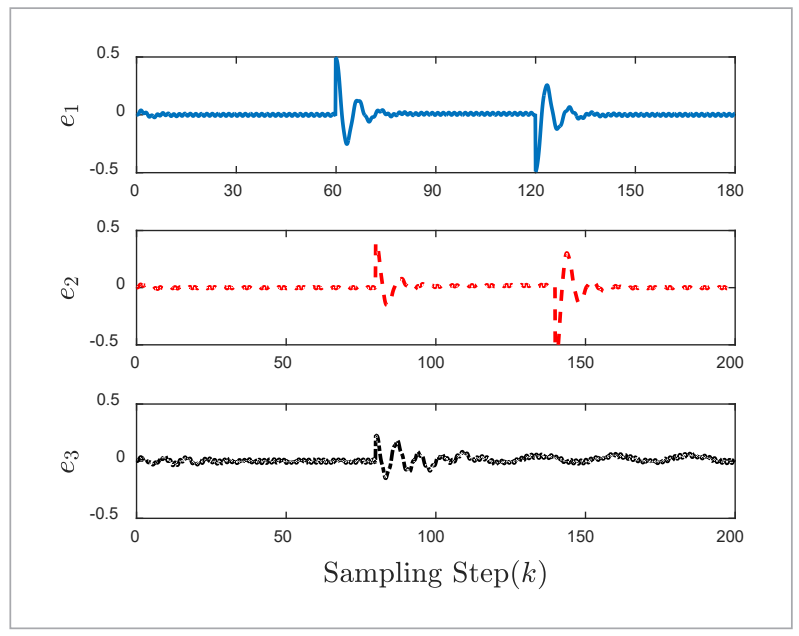

tiveness of the proposed scheme. Table 1 presents the simulation errors for the reconstruction of three component faults from a quantitative perspective, while $E_{\max }, E_{\text {ave }}$ and $S t d$ represent the max value, average value and standard deviation of the errors. The average error is within the allowable range, while the standard deviation meets the real requirements.
Table 1

Simulation errors for three component faults

\begin{tabular}{c|c|c|c}
\hline Error & $E_{\max }$ & $E_{\text {ave }}$ & $S t d$ \\
\hline$e_{1}$ & 0.4902 & 0.0031 & 0.0641 \\
\hline$e_{2}$ & 0.3944 & 0.0042 & 0.0706 \\
\hline$e_{3}$ & 0.2248 & 0.0119 & 0.0361 \\
\hline
\end{tabular}

The first actuator is on normal running with $\bar{f}(k)=1$ for all the time. Figure 6 represents the influence of LOs on the normal actuator, where one can see that the fault reconstruction for a faulty actuator will affect another normal one. However, the influence is within the system permissibility.

\section{Figure 6}

The effect on normal actuator

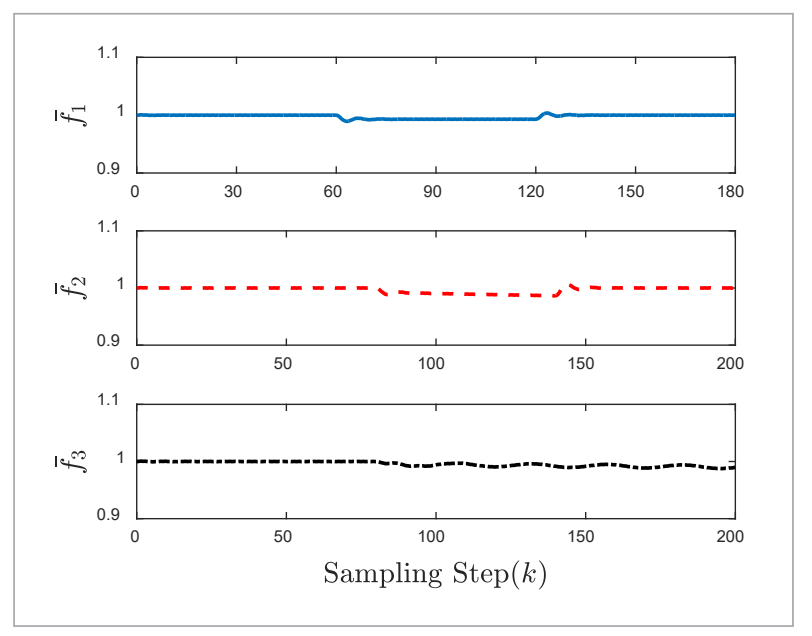

$K_{1}$ plays an important role of "Memory" for the reconstructed fault signal. In general, $K_{1}$ is a diagonal matrix with elements close to one. One can determine the value of $K_{1}$ based on the degree of fluctuation of fault signals. A relatively smaller value of $K_{1}$ can help the LOs easily ignore the historical signal and keep track of the fault changes. A simulation experiment to verify the influence of $K_{1}$ is established subject to $\tilde{f}_{1}(k)$. Three values are assigned to $K_{1}: k_{1}=0.92 I, k_{2}=0.95 I, \quad k_{3}=0.98 I$. Figure 7 presents the simulation results. $\tilde{f}_{1}(k)$ has relatively large changes at the sampling point $k=60$ and $k=120$. Based on the selection criteria of the value of $K_{1}$, one should choose a relatively smaller value 
of $K_{1}$, which can easily ignore the histrorical signal and keep track of the fault changes. Figure 7 shows that three simulation results all converge to the true fault signal, and the first $k_{1}$ value is the best choice with relatively small fluctuations.

\section{Figure 7}

Simulation results for $\tilde{f}_{1}(k)$ based on different $K_{1}$

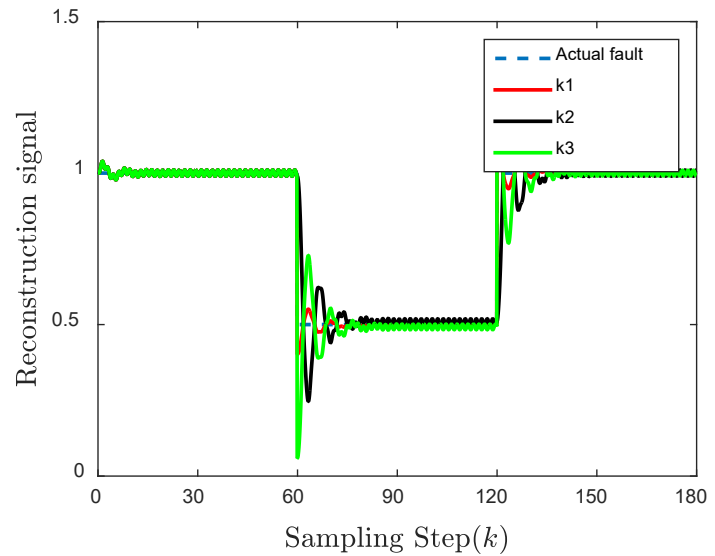

Figure 8

Simulation results for $\tilde{f}_{3}(k)$ with external noise

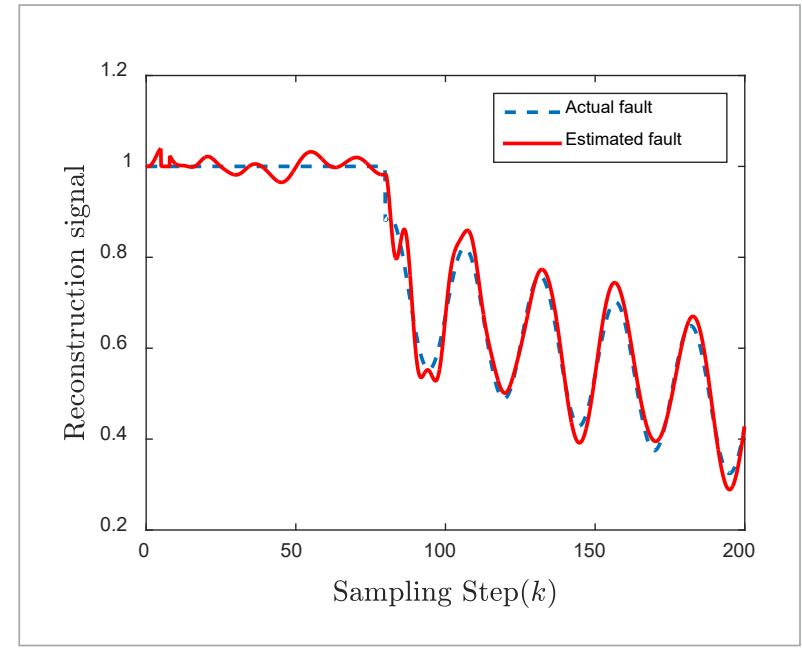

In addition, in order to verify the robustness of the proposed component fault diagnosis scheme, an external disturbance $\eta_{k}$ is added in system (9). There are two actuators in the simulation system. We consider two diffenret external noises are as follows:

$$
\eta_{k}=\left[\begin{array}{c}
0.02 * \sin (3.8 * k / 10) \\
0.01 * \sin (2.5 * k / 10)+0.01 * \cos (k / 8)
\end{array}\right] .
$$

Figure 8 presents the simulation result for $\tilde{f}_{3}(k)$ with the external noise. Comparied with the simulation result for $\tilde{f}_{3}(k)$ without external noise in Figure 4 , there exist some fluctuations for the output of the LOs during the first 100 steps. Then it tends to vary gently. It can also well reconstruct the component faults under the disturbance of external noise.

\section{Conclusion}

There are relatively few research works for the component fault diagnosis of switched system. In this paper, an active fault detection and estimation scheme based on LOs is investigated against component faults in a class of switched systems. The proposed LOs can guarantee the uniformly ultimately boundedness of fault reconstruction error with external disturbance using switched Lyapunov function and $H_{\infty}$ technique. The design of the LOs is simple and easy to implement, which can deal with different types of fault. In the simulation parts, three kinds of fault reconstruction examples have demonstrated the effectiveness of the proposed method.

The research of this paper is a supplement to the field of fault diagnosis of switched systems. It can also be extended to other control systems. Future works are as follows:

1 The system involved in this paper is a linear switched system. However, most switched systems exhibit nonlinear characteristics. It is necessary to consider the effects of nonlinearity.

2 The choice of $K_{1}$ and $\tau$ in the LOs are generally based on human experience. It should be designed an adaptive change rule for the parameters in the LOs.

\section{Appendix}

There are three parts in the appendix for the proofs of the theorems presented above.

Part A: Proof for Theorem 1: First two lemmas are listed which are useful in the following proof.

Lemma 1 [24]: For a positive defined symmetric ma- 
trix $P$, and two arbitrary matrices $M$ and $N$ with appropriate dimensions, the following inequality holds:

$$
2 M P N \leq \gamma M P M^{T}+N^{T} P N / \gamma,
$$

where $\gamma>0$.

Lemma 2 [13, 21]: For the fault updating observer established in (4), the following inequality for fault reconstruction error is satisfied

$$
\begin{aligned}
& e_{F}^{T}(k) e_{F}(k) \leq \rho_{1} e_{F}^{T}(k-\tau) K_{1}^{T} K_{1} e_{F}(k-\tau) \\
& \quad+\rho_{2} e_{x}^{T}(k)\left(K_{2 i} C_{i}\right)^{T}\left(K_{2 i} C_{i}\right) e_{x}(k)+\rho_{3} \tilde{F}_{k}^{T} \tilde{F}_{k},
\end{aligned}
$$

where $\quad \rho_{1}=1+\vartheta_{1}+\vartheta_{2}, \quad \rho_{2}=1+1 / \vartheta_{1}+\vartheta_{3} \quad$ and $\rho_{3}=1+1 / \vartheta_{2}+1 / \vartheta_{3}$ with $\vartheta_{i}>0, i=1,2,3$.

Consider the following Lyapunov function

$$
\begin{aligned}
V(k)= & e_{x}^{T}(k)\left(\sum_{i=1}^{N} \xi_{i}(k) P_{i}\right) e_{x}(k) \\
& +\sum_{j=k-\tau}^{k-1} e_{F}^{T}(j)\left(\sum_{i=1}^{N} \xi_{i}(j) Q_{i}\right) e_{F}(j)
\end{aligned}
$$

where $P_{i}$ and $Q_{i}$ are the positive defined symmetric matrices. Then the difference of Lyapunov function is expressed as

$$
\begin{aligned}
& \Delta V(k)=V(k+1)-V(k) \\
& =e_{x}^{T}(k+1)\left(\sum_{i=1}^{N} \xi_{i}(k+1) P_{i}\right) e_{x}(k+1) \\
& -e_{x}^{T}(k)\left(\sum_{i=1}^{N} \xi_{i}(k) P_{i}\right) e_{x}(k) \\
& +e_{F}^{T}(k)\left(\sum_{i=1}^{n} \xi_{i}(k) Q_{i}\right) e_{F}(k) \\
& -e_{F}^{T}(k-\tau)\left(\sum_{i=1}^{N} \xi_{i}(k-\tau) Q_{i}\right) e_{F}(k-\tau)
\end{aligned}
$$

The difference equation (20) above corresponds to any mode of switching rules. As declared in Assumption 1 , only one subsystem is active each time. So we assume $\xi_{i}(k)=1, \quad \xi_{\varphi \neq i}(k)=0, \quad \xi_{j}(k+1)=1$, $\xi_{\varphi \neq j}(k+1)=0, \quad \xi_{l}(k-\tau)=1, \quad \xi_{\varphi \neq l}(k-\tau)=0$. Then formula (20) can be simplified into

$$
\begin{gathered}
\Delta V(k)=e_{x}^{T}(k+1) P_{j} e_{x}(k+1)-e_{x}^{T}(k) P_{i} e_{x}(k) \\
+e_{F}^{T}(k) Q_{i} e_{F}(k)-e_{F}^{T}(k-\tau) Q_{l} e_{F}(k-\tau) \\
=e_{x}^{T}(k)\left[\left(A_{i}-L_{i} C_{i}\right)^{T} P_{j}\left(A_{i}-L_{i} C_{i}\right)-P_{i}\right] e_{x}(k) \\
+2 e_{x}^{T}(k)\left(A_{i}-L_{i} C_{i}\right)^{T} P_{j} U_{k} e_{F}(k) \\
-e_{F}^{T}(k-\tau) Q_{l} e_{F}(k-\tau) \\
+e_{F}^{T}(k)\left[U_{k}^{T} P_{j} U_{k}+Q_{i}\right] e_{F}(k)
\end{gathered} .
$$

According to Lemma 1, one can get

$$
\begin{aligned}
& 2 e_{x}^{T}(k)\left(A_{i}-L_{i} C_{i}\right)^{T} P_{j} U_{k} e_{F}(k) \leq \\
& \gamma_{1} e_{x}^{T}(k)\left(A_{i}-L_{i} C_{i}\right)^{T} P_{j}\left(A_{i}-L_{i} C_{i}\right) e_{x}(k), \\
& +1 / \gamma_{1} e_{F}^{T}(k) U_{k}^{T} P_{j} U_{k} e_{F}(k)
\end{aligned}
$$

where $M=e_{x}^{T}(k)\left(A_{i}-L_{i} C_{i}\right)^{T}, \quad P=\left(A_{i}-L_{i} C_{i}\right)^{T}$ and $N=U_{k} e_{F}(k)$.

Based on Assumption 2, the formula (21) satisfies the following inequality

$$
\begin{aligned}
\Delta V(k) & \leq e_{x}^{T}(k)\left[\begin{array}{l}
\left(1+\gamma_{1}\right)\left(A_{i}-L_{i} C_{i}\right)^{T} P_{j} \\
\cdot\left(A_{i}-L_{i} C_{i}\right)-P_{i}
\end{array}\right] e_{x}(k) \\
& -\delta e_{F}^{T}(k) Q_{i} e_{F}(k)+\alpha e_{F}^{T}(k) e_{F}(k) \\
& -e_{F}^{T}(k-\tau) Q_{l} e_{F}(k-\tau)
\end{aligned},
$$

where $\alpha=\left(1+1 / \gamma_{1}\right) \lambda_{\max }\left(P_{j}\right) u_{\max }^{2}+(1+\delta) \lambda_{\max }\left(Q_{l}\right)$ with $\delta \geq 0$.

Applying Lemma 2 , the above inequality can be further expressed as

$$
\begin{aligned}
& \Delta V(k) \leq e_{x}^{T}(k)\left[\left(1+\gamma_{1}\right)\left(A_{i}-L_{i} C_{i}\right)^{T} P_{j}\left(A_{i}-L_{i} C_{i}\right)\right. \\
& \left.-P_{i}+\alpha \rho_{2}\left(K_{2 i} C_{i}\right)^{T}\left(K_{2 i} C_{i}\right)\right] e_{x}(k)-\delta e_{F}^{T}(k) Q_{i} e_{F}(k) . \quad \text { (24) } \\
& +e_{F}^{T}(k-\tau)\left(\alpha \rho_{1} K_{1}^{T} K_{1}-Q_{l}\right) e_{F}(k-\tau)+\alpha \rho_{3} \tilde{F}_{k}^{T} \tilde{F}_{k}
\end{aligned}
$$

From inequality (7) with Schur complement lemma, one can get

$$
\begin{aligned}
\left(A_{i}\right. & \left.-L_{i} C_{i}\right)^{T} P_{j}\left(A_{i}-L_{i} C_{i}\right)-P_{i} \\
& +\alpha \rho_{2}\left(K_{2 i} C_{i}\right)^{T}\left(K_{2 i} C_{i}\right)<0
\end{aligned}
$$


Let $\Lambda$ represent the left-hand side of the above inequality, and define $\lambda_{1}=\lambda_{\min }(-\Lambda)>0$ and $\lambda_{2}=\delta \lambda_{\min }\left(-Q_{i}\right)>0$. Together with Assumption 3, one can get

$$
\Delta V(k) \leq-\lambda_{1}\left\|e_{x}(k)\right\|^{2}-\lambda_{2}\left\|e_{F}(k)\right\|^{2}+\alpha \rho_{3} \kappa_{f}^{2} .
$$

Thus

$$
\max \left[\left\|e_{x}(k)\right\|,\left\|e_{F}(k)\right\|\right] \leq \sqrt{\frac{\alpha \rho_{3}}{\lambda_{1}+\lambda_{2}}} \kappa_{f} .
$$

Hence, the uniform ultimate boundedness of $e_{x}(k)$ and $e_{F}(k)$ can be guaranteed.

Part B: Proof for Theorem 2: Consider the following Lyapunov function:

$$
V(k)=V_{1}(k)+V_{2}(k)+V_{3}(k)+V_{4}(k),
$$

where

$$
\begin{aligned}
& V_{1}(k)=e_{x}^{T}(k)\left(\sum_{i=1}^{N} \xi_{i}(k) P_{i}\right) e_{x}(k) \\
& V_{2}(k)=\sum_{j=k-d_{k}}^{k-1} e_{x}^{T}(j)\left(\sum_{i=1}^{N} \xi_{i}(j) Q_{i}\right) e_{x}(j) \\
& V_{3}(k)=\sum_{j=k-\bar{d}}^{k-d} \sum_{s=j}^{k-1} e_{x}^{T}(s)\left(\sum_{i=1}^{N} \xi_{i}(s) Q_{i}\right) e_{x}(s) . \\
& V_{4}(k)=\sum_{j=k-\tau}^{k-1} e_{F}^{T}(j)\left(\sum_{i=1}^{N} \xi_{i}(j) R_{i}\right) e_{F}(j)
\end{aligned}
$$

Then

$$
\begin{aligned}
\Delta V_{1}(k)= & e_{x}^{T}(k+1) P_{j} e_{x}(k+1) \\
& -e_{x}^{T}(k) P_{i} e_{x}(k)
\end{aligned},
$$

$$
\begin{aligned}
& \Delta V_{2}(k)+\Delta V_{3}(k) \leq \sigma e_{x}^{T}(k) Q_{i} e_{x}(k) \\
& -e_{x}^{T}(k-\underline{d}) Q_{k-\underline{d}} e_{x}(k-\underline{d}) \\
& -e_{x}^{T}(k-\underline{d}) Q_{k-\underline{d}} e_{x}(k-\underline{d}) \\
& -e_{x}^{T}(k-\bar{d}) Q_{k-\bar{d}} e_{x}(k-\bar{d})
\end{aligned}
$$

$$
\begin{aligned}
\Delta V_{4}(k)= & e_{F}^{T}(k) R_{i} e_{F}(k) \\
& -e_{F}^{T}(k-\tau) R_{l} e_{F}(k-\tau),
\end{aligned}
$$

where $\sigma=(\bar{d}-\underline{d}+2)$. Then setting $\lambda_{\min }(Q)=\min \left\{\lambda_{\min }\left(Q_{j}\right), j=k-\underline{d}, k-\underline{d}-1, \cdots, k-\bar{d}\right\}, \quad$ or $\lambda_{\text {min }}(Q)=\min \left\{\lambda_{\text {min }}\left(Q_{i}\right), i \in \aleph\right\}$, one can get

$$
\begin{aligned}
& \Delta V_{2}(k)+\Delta V_{3}(k) \leq \sigma e_{x}^{T}(k) Q_{i} e_{x}(k) \\
& -\lambda_{\min }(Q) e_{x}^{T}\left(k-d_{k}\right) e_{x}\left(k-d_{k}\right)
\end{aligned}
$$

Combining the state estimation error in (11), one can get

$$
\begin{aligned}
S= & e_{x}^{T}(k+1) P_{j} e_{x}(k+1) \\
= & e_{x}^{T}(k)\left(A_{i}-L_{i} C_{i}\right)^{T} P_{j}\left(A_{i}-L_{i} C_{i}\right) e_{x}(k) \\
& +e_{x}^{T}\left(k-d_{k}\right) A_{d i}^{T} P_{j} A_{d i} e_{x}\left(k-d_{k}\right) \\
& +e_{F}^{T}(k) U_{k}^{T} P_{j} U_{k} e_{F}(k)+\eta_{k}^{T} E_{i}^{T} P_{j} E_{i} \eta_{k} \\
& +2 e_{x}^{T}(k)\left(A_{i}-L_{i} C_{i}\right)^{T} P_{j} A_{d i} e_{x}\left(k-d_{k}\right) \\
& +2 e_{x}^{T}(k)\left(A_{i}-L_{i} C_{i}\right)^{T} P_{j} U_{k} e_{F}(k) \\
& +2 e_{x}^{T}(k)\left(A_{i}-L_{i} C_{i}\right)^{T} P_{j} E_{i} \eta_{k} \\
& +2 e_{x}^{T}\left(k-d_{k}\right) A_{d i}^{T} P_{j} U_{k} e_{F}(k) \\
& +2 e_{x}^{T}\left(k-d_{k}\right) A_{d i}^{T} P_{j} E_{i} \eta_{k}+2 e_{F}^{T}(k) U_{k}^{T} P_{j} E_{i} \eta_{k}
\end{aligned}
$$

We employ Lemma 1 to simplify the above equation. For the term $2 e_{x}^{T}(k)\left(A_{i}-L_{i} C_{i}\right)^{T} P_{j} A_{d i} e_{x}\left(k-d_{k}\right)$, one can define $M=e_{x}^{T}(k)\left(A_{i}-L_{i} C_{i}\right)^{T}, N=A_{d i} e_{x}\left(k-d_{k}\right)$, $P=P_{j}$, then

$$
\begin{aligned}
& 2 e_{x}^{T}(k)\left(A_{i}-L_{i} C_{i}\right)^{T} P_{j} A_{d i} e_{x}\left(k-d_{k}\right) \leq \\
& \gamma_{1} e_{x}^{T}(k)\left(A_{i}-L_{i} C_{i}\right)^{T} P_{j}\left(A_{i}-L_{i} C_{i}\right) e_{x}(k), \\
& +1 / \gamma_{1} e_{x}^{T}\left(k-d_{k}\right) A_{d i}^{T} P_{j} A_{d i} e_{x}\left(k-d_{k}\right)
\end{aligned}
$$

where $\gamma_{1}>0$.

Similarly, one can get

$$
\begin{aligned}
& 2 e_{x}^{T}(k)\left(A_{i}-L_{i} C_{i}\right)^{T} P_{j} U_{k} e_{F}(k) \leq \\
& \quad \gamma_{2} e_{x}^{T}(k)\left(A_{i}-L_{i} C_{i}\right)^{T} P_{j}\left(A_{i}-L_{i} C_{i}\right) e_{x}(k) \\
& \quad+1 / \gamma_{2} e_{F}^{T}(k) U_{k}^{T} P_{j} U_{k} e_{F}(k)
\end{aligned}
$$




$$
\begin{aligned}
& 2 e_{x}^{T}(k)\left(A_{i}-L_{i} C_{i}\right)^{T} P_{j} E_{i} \eta_{k} \leq \\
& \quad \gamma_{3} e_{x}^{T}(k)\left(A_{i}-L_{i} C_{i}\right)^{T} P_{j}\left(A_{i}-L_{i} C_{i}\right) e_{x}(k) \\
& \quad+1 / \gamma_{3} \eta_{k}^{T} E_{i}^{T} P_{j} E_{i} \eta_{k}
\end{aligned}
$$

$$
2 e_{x}^{T}\left(k-d_{k}\right) A_{d i}^{T} P_{j} U_{k} e_{F}(k) \leq
$$$$
\gamma_{4} e_{x}^{T}\left(k-d_{k}\right) A_{d i}^{T} P_{j} A_{d i} e_{x}\left(k-d_{k}\right)
$$$$
+1 / \gamma_{4} e_{F}^{T}(k) U_{k}^{T} P_{j} U_{k} e_{F}(k)
$$

$$
\begin{gathered}
2 e_{F}^{T}(k) U_{k}^{T} P_{j} E_{i} \eta_{k} \leq \gamma_{5} \eta_{k}^{T} E_{i}^{T} P_{j} E_{i} \eta_{k} \\
+1 / \gamma_{5} e_{F}^{T}(k) U_{k}^{T} P_{j} U_{k} e_{F}(k)
\end{gathered}
$$

where $\gamma_{1}, \gamma_{2}, \gamma_{3}, \gamma_{4}, \gamma_{5}>0$.

Based on Assumption 2, we can get

$$
\begin{aligned}
& S \leq\left[\begin{array}{l}
e_{x}^{T}(k)\left(1+\gamma_{1}+\gamma_{2}+\gamma_{3}\right)\left(A_{i}-L_{i} C_{i}\right)^{T} \cdot \\
P_{j}\left(A_{i}-L_{i} C_{i}\right) e_{x}(k)
\end{array}\right] \\
& +e_{x}^{T}\left(k-d_{k}\right)\left(1+1 / \gamma_{1}+\gamma_{4}\right) A_{d i}^{T} P_{j} A_{d i} e_{x}\left(k-d_{k}\right) \\
& +e_{F}^{T}(k)\left(1+1 / \gamma_{2}+1 / \gamma_{4}+1 / \gamma_{5}\right) u_{\max }^{2} P_{j} e_{F}(k) \\
& +\eta_{k}^{T}\left(1+1 / \gamma_{3}+\gamma_{5}\right) E_{i}^{T} P_{j} E_{i} \eta_{k} \\
& +2 e_{x}^{T}\left(k-d_{k}\right) A_{d i}^{T} P_{j} E_{i} \eta_{k}
\end{aligned}
$$

Thus

$$
\begin{aligned}
& \Delta V(k) \leq e_{x}^{T}(k)\left[\begin{array}{l}
\left(1+\gamma_{1}+\gamma_{2}+\gamma_{3}\right)\left(A_{i}-L_{i} C_{i}\right)^{T} \\
\cdot P_{j}\left(A_{i}-L_{i} C_{i}\right)+\sigma Q_{i}-P_{i}
\end{array}\right] e_{x}(k) \\
& +e_{x}^{T}\left(k-d_{k}\right)\left[\begin{array}{l}
\left(1+1 / \gamma_{1}+\gamma_{4}\right) A_{d i}^{T} \cdot P_{j} A_{d i} \\
-\lambda_{\min }(Q)
\end{array}\right] e_{x}\left(k-d_{k}\right) \\
& +\eta_{k}^{T}\left(1+1 / \gamma_{3}+\gamma_{5}\right) E_{i}^{T} P_{j} E_{i} \eta_{k}-\delta e_{F}^{T}(k) R_{i} e_{F}(k) \\
& +\alpha e_{F}^{T}(k) e_{F}(k)-e_{F}^{T}(k-\tau) R_{l} e_{F}(k-\tau) \\
& +2 e_{x}^{T}\left(k-d_{k}\right) A_{d i}^{T} P_{j} E_{i} \eta_{k}
\end{aligned}
$$

where $\alpha=\left(1+1 / \gamma_{2}+1 / \gamma_{4}+1 / \gamma_{5}\right) u_{\max }^{2} \lambda_{\max }\left(P_{j}\right)+(1+\delta) \lambda_{\max }\left(R_{i}\right)$. Using Lemma 2 , we obtain

$$
\begin{aligned}
& \Delta V(k) \leq e_{x}^{T}(k)\left[\begin{array}{l}
\zeta_{1}\left(A_{i}-L_{i} C_{i}\right)^{T} P_{j} \cdot\left(A_{i}-L_{i} C_{i}\right) \\
+\sigma Q_{i}-P_{i} \\
+\alpha \rho_{2}\left(K_{2 i} C_{i}\right)^{T}\left(K_{2 i} C_{i}\right)
\end{array}\right] e_{x}(k) \\
& \quad+e_{F}^{T}(k-\tau)\left(\alpha \rho_{1} K_{1}^{T} K_{1}-R_{l}\right) e_{F}(k-\tau) \\
& \quad-\delta e_{F}^{T}(k) R_{i} e_{F}(k)+\zeta_{3} \eta_{k}^{T} E_{i}^{T} P_{j} E_{i} \eta_{k} \\
& \quad+e_{x}^{T}\left(k-d_{k}\right)\left[\zeta_{2} A_{d i}^{T} P_{j} A_{d i}-\lambda_{\min }(Q)\right] e_{x}\left(k-d_{k}\right) \\
& \quad+2 e_{x}^{T}\left(k-d_{k}\right) A_{d i}^{T} P_{j} E_{i} \eta_{k}+\alpha \rho_{3} \tilde{F}_{k}^{T} \tilde{F}_{k}
\end{aligned}
$$

where $\zeta_{1}=1+\gamma_{1}+\gamma_{2}+\gamma_{3}, \quad \zeta_{2}=1+1 / \gamma_{1}+\gamma_{4} \quad$ and
$\zeta_{3}=1+1 / \gamma_{3}+1 / \gamma_{5}$. In order to make the proposed LOs robust to external disturbance, consider the following $H_{\infty}$ performance index

$$
J=\sum_{k=0}^{K}\left[e_{y}^{T}(k) e_{y}(k)-\gamma^{2} \eta_{k}^{T} \eta_{k}\right],
$$

where $K$ is an arbitrary positive integer.

Under zero-initial conditions, one has

$$
\begin{aligned}
J & =\sum_{k=0}^{K}\left[e_{y}^{T}(k) e_{y}(k)-\gamma^{2} \eta_{k}^{T} \eta_{k}+\Delta V(k)\right]-V(K) \\
& \leq \sum_{k=0}^{K}\left[e_{y}^{T}(k) e_{y}(k)-\gamma^{2} \eta_{k}^{T} \eta_{k}+\Delta V(k)\right]
\end{aligned} .
$$

It is noted that

$$
\begin{aligned}
& e_{y}^{T}(k) e_{y}(k)-\gamma^{2} \eta_{k}^{T} \eta_{k}+\Delta V(k) \leq e_{x}^{T}(k) \Xi_{1} e_{x}(k) \\
& +e_{F}^{T}(k-\tau) \Xi_{3} e_{F}(k-\tau)+\alpha \rho_{3} \tilde{F}_{k}^{T} \tilde{F}_{k} \\
& +\left[e_{x}^{T}\left(k-d_{k}\right) \eta_{k}^{T}\right] \Xi_{2}\left[\begin{array}{c}
e_{x}\left(k-d_{k}\right) \\
\eta_{k}
\end{array}\right]
\end{aligned}
$$

Based on Theorem 2 together with Schur complement lemma, one can get $\Xi_{1}<0, \Xi_{2}<0, \Xi_{3}<0$. Thus

$$
\sum_{k=0}^{K}\left[e_{y}^{T}(k) e_{y}(k)-\gamma^{2} \eta_{k}^{T} \eta_{k}\right] \leq \sum_{k=0}^{K} \alpha \rho_{3} \tilde{F}_{k}^{T} \tilde{F}_{k} .
$$

Thus, the uniform ultimate boundedness of system state estimation error and fault reconstruction error are guaranteed and output estimation error satisfies $\left\|e_{y}(k)\right\| \leq \gamma \bar{\eta}+\sqrt{\alpha \rho_{3}} \kappa_{f}$. 
Part C: Proof for Theorem 3: Here we give the proof that LMI (12) is equivalent to the formula (15). Similarly, LMI (13) can be transformed into the inequality (16).

Assume that (15) is feasible. Then $G_{i}+G_{i}^{T}-P_{j}>0$ which means that $G_{i}$ is nonsingular. For strictly positive matrix $P_{j}$, one can get

$$
\left(P_{j}-G_{i}\right)^{T} P_{j}^{-1}\left(P_{j}-G_{i}\right) \geq 0 .
$$

Thus

$$
G_{i}^{T} P_{j}^{T} G_{i} \geq G_{i}+G_{i}^{T}-P_{j}
$$

Then (15) can be transformed into

\section{References}

1. Bao, L. P., Fei, S. M., Yue, L. Exponential Stability of Linear Distributed Parameter Switched Systems with Time-Delay. Journal of Systems Science and Complexity, 2014, 27(2), 263-275. https://doi.org/10.1007/ s11424-014-30'70-4

2. Benzaouia, A., Ouladsine, M., Naamane, A., Ananou, B. Fault Detection for Uncertain Delayed Switching Discrete-Time Systems. International Journal of Innovative Computing, Information and Control, 2012, 8(12), 8049-8062.

3. Blanke, M., Kinnaert, M., Lunze, J., Staroswiecki, M. Diagnosis and Fault-Tolerant Control. Springer, Berlin, 2006.

4. Branicky, M. S. Multiple Lyapunov Functions and Other Analysis Tools for Switched and Hybrid Systems. IEEE Transactions on Automatic Control, 1998, 43(4), 475482. https://doi.org/10.1109/9.664150

5. Chen, W., Saif, M. An Iterative Learning Observer for Fault Detection and Accommodation in Nonlinear Time-Delay Systems. International Journal of Robust and Nonlinear Control, 2006, 16(1), 1-19. https://doi. org/10.1002/rnc.1033

6. Chiang, M. L., Hsieh, H. C. A New Approach to the Fault Detection Problem for Mobile P2P Network. Information Technology and Control, 2012, 41(2), 151-161. http://dx.doi.org/10.5755/ j01.itc.41.2.913

$$
\left[\begin{array}{ccccc}
-\zeta_{1} G_{i}^{T} P_{j}^{-1} G_{i} & 0 & 0 & G_{i}^{T} A_{i}-Y_{i} C_{i} & I \\
* & -G_{i}^{T} P_{i} G_{i} & M_{i} C_{i} & 0 & G_{i} \\
* & * & -I & C & 0 \\
* & * & * & -\alpha \rho_{2} I & 0 \\
* & * & * & * & -\sigma G_{i}^{T} Q_{i}^{-1} G_{i}
\end{array}\right]<0 .
$$

Then, pre multiplying inequality (47) by $\operatorname{diag}\left\{G_{i}^{-T}, I, I, I, G_{i}^{-T}\right\}$ and post multiplying by $\operatorname{diag}\left\{G_{i}^{-1}, I, I, I, G_{i}^{-1}\right\}$, one can obtain (12). So the proof is finished and the parameters of LOs are calculated by $L_{i}=G_{i}^{-T} Y_{i}$ and $K_{2 i}=G_{i}^{-T} M_{i}$.

\section{Acknowledgement}

This work was supported by the National Natural Science Foundation of China (No. 61806208, 61502498) and Fundamental Research Funds for the Central Universities (No. 3122017001, 3122017011).

7. Daafouz, J., Riedinger, P., Iung, C. C. Stability Analysis and Control Synthesis for Switched Systems: A Switched Lyapunov Function Approach. IEEE Transactions on Automatic Control, 2002, 47(11), 1883-1887. https://doi.org/10.1109/TAC.2002.804474

8. Du, D. S., Bin, J., Shi, P., Karimi, H. R. Fault Detection for Continuous-time Switched Systems Under Asynchronous Switching. International Journal of Robust and Nonlinear Control, 2014, 24(11), 1694-1706. https://doi. org/10.1002/rnc. 2961

9. Hakem, A., Pekpe, K. M., Cocquempot, V. A Parameter-Free Method for Sensor Fault Detection and Isolation in Bilinear Systems. Proceedings of 2012 UKACC International Conference on Control, Cardiff, UK, September 3-5, 2012, 437-442. https://doi.org/10.1109/ CONTROL. 2012.6334670

10. He, D., Xi, C., Lu, A. Fault Detection for Switched T-S Fuzzy Systems in Finite Frequency Domain. Advances in Difference Equations, 2016, 62. https://doi. org/10.1186/s13662-016-0785-77

11. Hespanha, J. P., Morse, A. S. Stability of Switched Systems with Average Dwell-Time. Proceedings of 38th IEEE Conference on Decision and Control, Phoenix, Arizona, USA, December 7-10, 1999, 2655-2660. https:// doi.org/10.1109/CDC.1999.831330

12. Hwang, I., Kim, S., Kim, Y., Seah, C. E. A Survey of Fault Detection, Isolation, and Reconfiguration Meth- 
ods. IEEE Transactions on Control Systems Technology, 2010, 18(3), 636-653. https://doi.org/10.1109/ TCST.2009.2026285

13. Jia, Q. X., Chen, W., Zhang, Y. C., Chen, X. Q. Robust Fault Reconstruction via Learning Observers in Linear Parameter-Varying Systems Subject to Loss of Actuator Effectiveness. IET Control Theory and Applications, 2014, 8(1), 42-50. https://doi.org/10.1049/iet-cta.2013. 0417

14. Jiang, B., Staroswiecki, M., Cocquempot, V. Fault Accommodation for Nonlinear Dynamic Systems. IEEE Transactions on Automatic Control, 2006, 51(9), 15781583. https://doi.org/10.1109/TAC. 2006.878732

15. Martins, R. S., Vale, M. R. B. G., Maitelli, A. L. Hybrid Methods for Detection and Identification of Faults in Dynamic Systems. Asian Journal of Control, 2015, 17(5), 1831-1847. https://doi.org/10.1002/asjc.1039

16. Rodrigues, M., Theilliol, D., Sauter, D. Fault Tolerant Control Design for Switched Systems. IFAC Proceedings Volumes, 2006, 39(5), 223-228. https://doi. org/10.3182/20060607-3-IT-3902.00041

17. Valipour, M. How Much Meteorological Information Is Necessary to Achieve Reliable Accuracy for Rainfall Estimations. Agriculture, 2016, 6(4), 1-9. https://doi. org/10.3390/ agriculture6040053

18. Valipour, M. Global Experience on Irrigation Management under Different Scenarios. Journal of Water and Land Development, 2017, 32(1), 95-102. https://doi. org/10.1515/jwld-2017-0011

19. Viero, D. P., Valipour, M. Modeling Anisotropy in Free-Surface Overland and Shallow Inundation Flows. Advances in Water Resources, 2017, 104, 1-14. https:// doi.org/10.1016/j.advwatres.2017. 03.007
20. Wang, D., Wang, W., Shi, P. Robust Fault Detection for Switched Linear Systems with State Delays. IEEE Transactions on Systems Man and Cybernetics, Part B, 2009, 39(3), 800-805. https://doi.org/10.1109/ TSMCB.2008.2007498

21. Xiang, M., Xiang, Z. R., Karimi, H. R. Stabilization of Positive Switched Systems with Time-Varying Delays under Asynchronous Switching. International Journal of Control, Automation, and Systems, 2014, 12(5), 939947. https://doi.org/10.1007/s12555-013-0486-x

22. Yu, J. Y., Liu, F. Z., Yu, X. X., Wu, C. F., Wu, L. G. Fault Detection of Discrete-time Switched Systems with Distributed Delays: Input-Output Approach. International Journal of Systems Science, 2013, 44(12), 2255-2272. https://doi.org/10.1080/00207721.2012.687786

23. Zhang, D., Yu, L., Zhang, W. A. Delay-Dependent Fault Detection for Switched Linear Systems with Time-varying Delays-the Average Dwell Time Approach. Signal Processing, 2011, 91(4), 832-840. https:// doi.org/10.1016/j.sigpro.2010.08.016

24. Zhang, F. Z. Matrix Theory. Springer, Berlin, 2011. https://doi.org/10.1007/978-1-4614-1099-7

25. Zhang, H. G., Zhang, S., Yin, Y. X. Adaptive Fault Diagnosis for Continuous Time-delay Repetitive System Subject to Sensor Fault. 2015 International Conference on Advanced Mechatronic Systems (ICAMechS), Beijing, China, August 22-24, 2015, 456-460. https://doi. org/10.1109/ ICAMechS.2015.7287154

26. Zhang, X. D., Polycarpou, M. M., Parisini, T. Fault Diagnosis of a Class of Nonlinear Uncertain Systems with Lipschitz Nonlinearities Using Adaptive Estimation. Automatica, 2010, 46(2), 290-299. https://doi. org/10.1016/j.automatica. 2009.11.014 